\title{
USABILITY EVALUATION OF PUBLIC WEB MAPPING SITES
}

\author{
WANG Cong \\ Department of TIANDITU, National Geomatics Center of China, No.28 Lianhuachi West Road, \\ Haidian District, Beijing, China \\ wangcong@nsdi.gov.cn
}

Key words: usability evaluation, public web mapping site, usability engineering

\begin{abstract}
:
Web mapping sites are interactive maps that are accessed via Webpages. With the rapid development of Internet and Geographic Information System (GIS) field, public web mapping sites are not foreign to people. Nowadays, people use these web mapping sites for various reasons, in that increasing maps and related map services of web mapping sites are freely available for end users. Thus, increased users of web mapping sites led to more usability studies. Usability Engineering (UE), for instance, is an approach for analyzing and improving the usability of websites through examining and evaluating an interface. In this research, UE method was employed to explore usability problems of four public web mapping sites, analyze the problems quantitatively and provide guidelines for future design based on the test results.
\end{abstract}

Firstly, the development progress for usability studies were described, and simultaneously several usability evaluation methods such as Usability Engineering (UE), User-Centered Design (UCD) and Human-Computer Interaction (HCI) were generally introduced. Then the method and procedure of experiments for the usability test were presented in detail. In this usability evaluation experiment, four public web mapping sites (Google Maps, Bing maps, Mapquest, Yahoo Maps) were chosen as the testing websites. And 42 people, who having different GIS skills (test users or experts), gender (male or female), age and nationality, participated in this test to complete the several test tasks in different teams. The test comprised three parts: a pretest background information questionnaire, several test tasks for quantitative statistics and progress analysis, and a posttest questionnaire. The pretest and posttest questionnaires focused on gaining the verbal explanation of their actions qualitatively. And the design for test tasks targeted at gathering quantitative data for the errors and problems of the websites. Then, the results mainly from the test part were analyzed. The success rate from different public web mapping sites was calculated and compared, and displayed by the means of diagram. And the answers from questionnaires were also classified and organized in this part. Moreover, based on the analysis, this paper expands the discussion about the layout, map visualization, map tools, search logic and etc. Finally, this paper closed with some valuable guidelines and suggestions for the design of public web mapping sites. Also, limitations for this research stated in the end.

\section{Introduction}

The Geographic Information System (GIS) field has experienced a significant development with the spread of World Wide Web (WWW). And the public web mapping sites are not unfamiliar for people any more, the increasing users inspired more attentions from researchers. Since the gaps between users and software developers always led to resources waste, the studies on usability becomes necessary. Usability engineering (UE) is a method to describe methods for analysing and enhancing the usability of software 
(Nielsen, 1994). Researchers generally used the UE method to study users' requirements and gained the guides for interactive maps. The User-Centred Design (UCD), however, is an approach throughout the entire life cycle of a system (ISO, 1999) and will achieve the needs of users. The process of UCD mostly can be seen as an iterative process. Moreover, the UCD usually referred to Human-Computer Interaction (HCI) methods. Both of them can help improve the usability of the interactive systems, so the methods above effects a positive role in usability studies of public web mapping sites. In this research, UE method and the user testing was employed to explore usability problems of four public web mapping sites, analyzed the problems quantitatively and provided guidelines for future design based on the test results.

\section{Methodology}

User testing was mainly used in this research which is a common method in HCI. Users (testing participants) would operate public web mapping sites to identify the errors and defects of them.

\subsection{Procedure}

Four public web mapping sites (Google Maps, Bing maps, Mapquest, Yahoo Maps) were chosen as the testing websites. And 42 people, who having different GIS skills (test users or experts), gender (male or female), age and nationality, participated in this test to complete the several test tasks in different teams. The small group of participants is recommended in usability literature (Noelsen, 1994). In this evaluation experiment, participants were required to complete certain operation tasks in a given scenario at the same time in a lab. The whole process of the test would be recorded by the computers.

\subsection{Description of the experiment}

The experiment comprised three parts: a pretest background information questionnaire, several test tasks for quantitative statistics and progress analysis, and a posttest questionnaire. Hence, the evaluation results should be both qualitative and quantitative information. The qualitative results are from the pretest and posttest questionnaires. The pretest questionnaire mainly get the basic and advanced information of each participant. The basic information is name, age, gender nationality, and the advanced information includes the description of GIS skills, the familiar level for the four map websites, the frequency of using map websites and etc. The purpose of the pretest is to get the background information. The posttest questionnaire aims at gaining the explanation for their operations and the visual feelings for the websites. Generally, the qualitative results will assist the researchers to understand the quantitative information from the experiment, and the verbal results is also the straightforward suggestions for the website designers.

However, the completion of test tasks may be the most important part of the experiment. In this research, participants would give a scenario to conduct the experiment step by step. The system will record the completion time of each task and the whole time of the experiment for each participant. The total clicks will also be recorded as the quantitative results. The three types of quantitative data can present the effective and efficient situation of the websites. And it can be also used in the success calculation for future study. The success rate is defined as "the percentage of tasks that users complete correctly" (Nielsen, 2001). In the process of completion, the failure operation will be scored as fail and the participants can continue the experiment for the next task. The success operation which gets the ideal results will be score as successful. Partial success operation will be given the weight of 0.5 . So the final success rate for each website can be calculated according to the formula (1):

$$
(\mathrm{Ns}(\mathrm{Nps} * 0.5)) / \mathrm{N}
$$

Where Ns: total number of successful operations

Nps:total Number of Partially Successful
operations
$\mathrm{N}$ : number of total operations

Moreover, the scenario of this experiment is that: complete the tasks as a traveler who arrived firstly in Washington, DC in USA. And the tasks designed to test the aspects of positioning, route planning, information query and website multi-functions for the map websites. And the general 
description of tasks listed below: (1) Open the website and change the needed language; (2) Locate the Scenic Spot (White House); (3) Find the other information for the White House such as scenic introduction, image picture, 3D model; (4) Search a desired hotel around and try to find other information about the hotel such as price and website link; (5) Find the route from the White House to your hotel, and choose a suitable route.

\section{Results}

\subsection{Quantitative Results}

The average time spend for each task of four websites is one part of quantitative data, and it is in strong correlation with the situation of usability for websites and the degree of the satisfaction. To some extent, the less time used to complete one task states that users achieve the goal with less attempts or good conditions of one websites in the aspects of task related. In the research, the results of the correlation between different web mapping sites and the average time spent (seconds) show in the Figure1 below.

As Figure 1 shows, different tasks led to the most different time spend. Complicated tasks would spend more time to achieve the goal. But for same tasks, different web mapping sites also have the different performance.

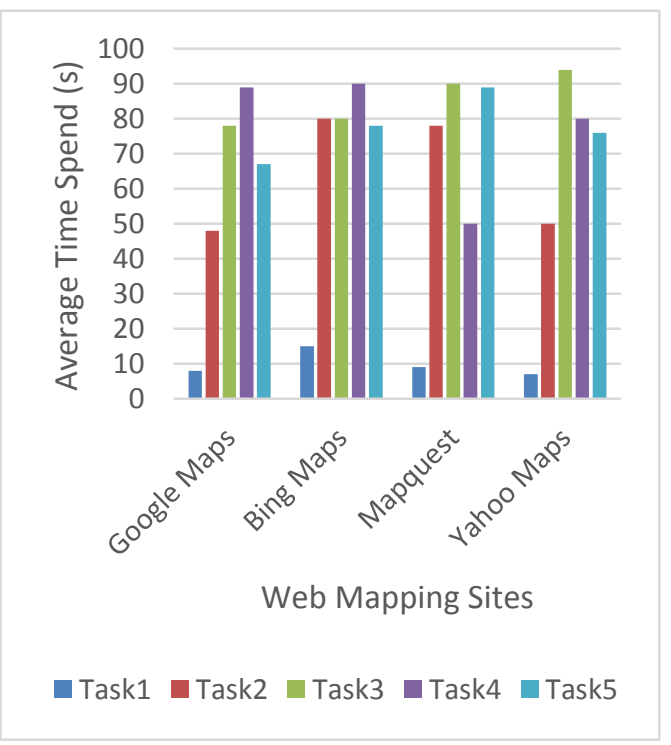

Figure1 Different Web Mapping Sites based on average time spend for each task in the experiment
Furthermore, based on the method of calculating success rate, the success rate of four web mapping sites has been recorded, calculated and displayed in Figure 2.

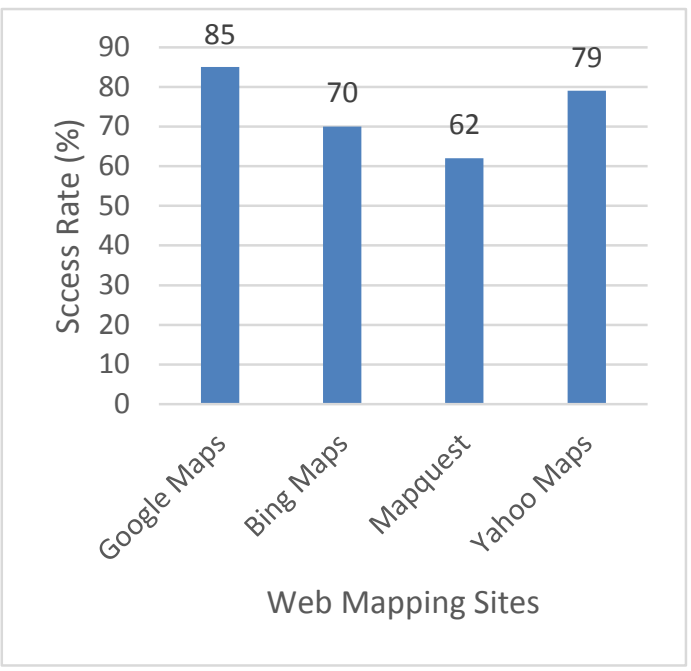

Figure 2 Success Rate (\%) for four web mapping sites

The success rate can explain the whole performance and usability condition directly and integrally. The Figure 2 indicates that, Google maps is the most suitable for participant to get the desirable results and can satisfy the participants' needs from the tasks above. And the less usable web mapping sites is Map Quest.

\subsection{Qualitative Results}

The qualitative results could be gathered in the questionnaire of pretest and posttest. And the posttest questionnaire is more valuable for identifying the errors and defects of web mapping sites. Participants explains their actions during the experiment and gives visualized description of the websites. These verbal data can be used to enhance the design of the websites directly for designers.

\section{Discussion}

The research can assist us evaluate the usability of the four web mapping sites. And the usability problems which occurred during the experiment give the designers suggestions and guidelines for future progress.

\subsection{User Interface}


The "start page" of web mapping sites is very important for users, for the reason that the first impression can guide the users' estimation for their "tasks". In many cases, too much information in the first page would make users feel messy and disordered, which may have a negative impact on their operations. In research, this problems may present in Task 1. Too many links, advertisements, and images may led to the low effective load speed. Users may spend more time to open the homepage, and wait for the whole information loading. Google maps is one example that included various information in the first page. The Yahoo Maps, however, has less information in the first page and the layout is very clean. Hence, for designers the design of the homepage should be clear and simple to let the users start using the map in a short time after entering the website page.

Moreover, the layout of the homepage should consider the user' usual habits. Some users cannot find the search box or location button when starting attempts. Too much time when spending on searching the button will led to the exhausted patience. For example, Map Quest is not clear for users to find the search box, and the "directions" and "locations" are difficult to get. Thus, the search box that users may be used firstly should be given an essential role in the layout. The search boxes which designed friendly and easily to use shows in Figure3. The two search boxes are from Map Quest and Yahoo Maps.

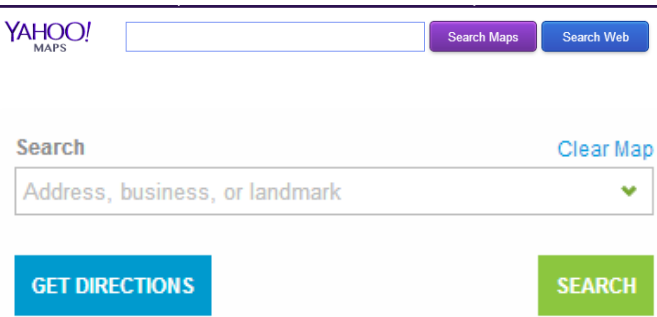

Figure 3 Different Types of Search Boxes

\subsection{Functionality}

In a way, functionality decided the performance of one web mapping site. All users want the web sites has a high feedback speed, so the rapid responding speed is vital when clicking the buttons on web mapping site. When conducting certain search query or other operations, a new page will appear out of the previous window. So this problem is that the "back-button" cannot guide the users back to the previous page or homepage and get lost in the sites. In the verbal questionnaire, users expect that there should be a short cut back to the home page to avoid lost when using the website functions such as route display, pictures of the POI and other advanced information. Thus when linking with more additional services (such as hotels and tourist attractions), the web mapping sites will led to the problems above.

\subsection{Search Operations}

Search Operations can mainly identify usability problems relating information queries. The search logic, default settings and the search results have an important effect on the using experience for users. From the posttest questionnaire, several suggestions have been proposed. 1) The box can be operated the search is single. Some users want to search more than one addresses or locations, but most of web mapping sites just provide just one search box. The "directions" cannot satisfy the users' needs, because sometimes they only want to get the spatial locations on one screen size. For example, some participants in the experiments want to search the "White House" and the hotel at the same time. But the four websites cannot allow this operation. 2) The default Settings also confused users sometimes. Some web mapping sites set the first page locating at the current location such as Bing Maps, users should change the location in USA then search the "White House" in the experiment. While some participants cannot find the "location changed" button easily. Also, some websites will change the language to the location, and the change button is also difficult to find. These default settings will make users feel boring when achieving their objectives. 3) The search results may be ultimate display of the search operation. But the display of the search results in some web mapping sites are messy. When searching certain location, the results are shown disordered. Take the "White House" as an example, in some websites, when finishing searching the "White House", results from different countries displayed. Users should choose the right one from several options. Thus, the information from the posttest questionnaire about search operation is valuable for the designers to enhance the search operation of the web mapping sites.

\subsection{Visualization}


The Visualization of the web mapping sites would be a complicated element for designers. There always a gap between the designers and the users. Some factors such as colour and fashion are difficult to judge. Some feedbacks from questionnaire show that visualization is restless and awful, and the colours may be old-fashioned. Despite this, this part is difficult to balance the needs of users and the ideas of designers.

This discussion proposed some guidelines for designers based on the experiments, but there still some limitations of this experiments, such as test tasks and questionnaire.

\section{Conclusion}

The usability evaluation studies are popular nowadays. In this paper, the experiment aimed at identifying usability problems and providing suggestions for designers. The problems and advices were proposed on the aspects of User Interface, functionality, search operations and visualization to enhance the performance of the websites.

\section{REFERENCE}

Annu-Maaria Nivala., 2008, Usability Evaluation of Web Mapping Sites. The Cartographic Journal 45 (2), pp.129-138 Bing Maps., 2014, online at: http://cn.bing.com/maps/ Google Maps., 2014, online at: https://maps.google.com/ ISO 13407(1999) 'Human-Centered Design for Interactive System', International Organisation for Standardisation, Geneva, Switzerland.

Map Quest., 2014, online at: http://www.mapquest.com/

Nielsen, J., 1994, Guerilla HCI: Using Discount Usability Engineering to Penetrate the Inimidation Barrier. Http://www.useit.com/papers/guerilla hci.html

Nielsen.J., 2001, Success Rate: The simplest usability Metric. Http://www.useit.com/alertbox/20010218.html

Yahoo Maps., 2014, online at: http://maps.yahoo.com/ 